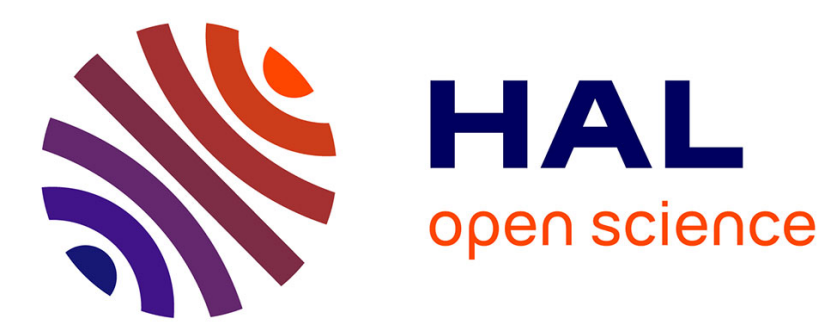

\title{
Les indicateurs de performance
}

Joëlle Morana, Jesus Gonzalez-Feliu

\section{To cite this version:}

Joëlle Morana, Jesus Gonzalez-Feliu. Les indicateurs de performance. 2010. halshs-01055895

\section{HAL Id: halshs-01055895 \\ https://shs.hal.science/halshs-01055895}

Submitted on 13 Aug 2014

HAL is a multi-disciplinary open access archive for the deposit and dissemination of scientific research documents, whether they are published or not. The documents may come from teaching and research institutions in France or abroad, or from public or private research centers.
L'archive ouverte pluridisciplinaire $\mathbf{H A L}$, est destinée au dépôt et à la diffusion de documents scientifiques de niveau recherche, publiés ou non, émanant des établissements d'enseignement et de recherche français ou étrangers, des laboratoires publics ou privés. 


\title{
Chapitre $X$ : Les indicateurs de performance
}

\author{
Joëlle Morana* Jesus Gonzalez-Feliu* \\ *LET, Université de Lyon \\ ISH - 14 Avenue Berthelot \\ 69363 Lyon cedex 07 \\ joelle.morana@let.ish-lyon.cnrs.fr \\ jesus.gonzales-feliu@let.ish-lyon.cnrs.fr
}

\section{Introduction}

La notion de performance s'est longuement placée dans une optique financière où la satisfaction des actionnaires (shareholders) est privilégiée. Toutefois, de plus en plus de recherches allient aux indicateurs financiers, des indicateurs non financiers. En fait, le but poursuivi est de concevoir une évaluation multi-critère et multi-dimensionnelle où les intérêts de tous les acteurs (stakeholders) sont intégrés (Kaplan et Norton, 1996 ; Bouquin, 1997 ; Bourrier et al., 1998).

Toutefois, là ne n'arrête pas cette évolution. En effet, l'incidence d'une logique de Développement Durable se fait sentir de manière de plus en plus prégnante au sein des entreprises. Selon cette visée, les entreprises doivent alors réfléchir à la pratique d'indicateurs de type économique, environnemental et social.

La problématique de l'évaluation de la logistique, et plus précisément de la logistique urbaine, est un élément essentiel qui transparaît au long de son évolution. Dans une optique de développement durable, les écrits montrent une réflexion sur la performance du Supply Chain Management (réflexion économique), du Green Supply Chain Management (réflexion environnementale) et du Social Supply Chain Management (réflexion sociale). En découle depuis ces dernières années à l'émergence d'un Sustainable Supply Chain Management dont le but est une conjugaison de ces trois aspects. De plus, les spécificités du dernier kilomètre urbain doivent aussi être prises en compte

Ce chapitre se déclinera tel que suit. Tout d'abord, un premier paragraphe présentera quelques 
travaux de référence sur la notion de performance, de même qu'un travail de synthèse d'indicateurs de performance. Ensuite, le deuxième paragraphe soulignera les travaux sur la mesure de la performance en lien avec la logistique. Enfin, le troisième paragraphe aura pour lieu de faire le point sur les indicateurs de performance durable de la logistique urbaine.

\section{La notion de performance et efficacité organisationnelle}

Selon Marmuse (1997), « la performance revêt (...) des aspects multiples, sans doute convergents, mais qui méritent d'être abordés dans une logique plus globale que la seule appréciation de la rentabilité pour l'entreprise ou pour l'actionnaire» (p. 2194). Ainsi, plusieurs aspects de la performance doivent être envisagés :

- tout d'abord, selon une dimension stratégique qui fédère les actions entreprises autour de la pérennité ;

- ensuite, sous l' angle de la performance concurrentielle qui consiste à rechercher des solutions au-delà d'une unidimensionnalité de la structure ;

- enfin, dans une perspective de performance socio-économique qui raisonne sur la reconfiguration interne des approches organisationnelles et sociales.

Selon une même hypothèse, il est possible de considérer le travail de Cross et Lynch (1989) comme une référence en l'état. Ces auteurs ont cherché, au travers de la pyramide des performances, à apporter une lecture utile dans un déploiement de la mesure de la performance. Pour cela, ils ont combiné le champ opérationnel et le champ stratégique pour illustrer la mise en œuvre d'indicateurs étroitement reliés au plus haut niveau de la structure (vision) jusqu'aux actions quotidiennes développées par l'entreprise (opérations) (Cross et Lynch, 1989).

En soi, l'objectif pour toute entreprise est donc d'améliorer son efficacité. A ce titre, le travail doctoral de Montebello (1976), quoique relativement ancien, constitue un apport indéniable dans la définition de l'efficacité organisationnelle. Celle-ci peut se définir selon sept angles d'attaque. Il s'agit :

(1) du degré de réalisation des objectifs,

(2) de l'efficience et de la productivité,

(3) de l'adaptation aux contraintes de l'environnement,

(4) de l'exploitation de l'environnement dans l'acquisition de ressources rares et de valeurs, 
(5) de la maximisation des retours à l'entreprise,

(6) de l'accomplissement de certaines exigences fonctionnelles et enfin,

(7) de la valeur sociale de l'entreprise.

Figure 1. La pyramide des performances selon Cross et Lynch (1989)

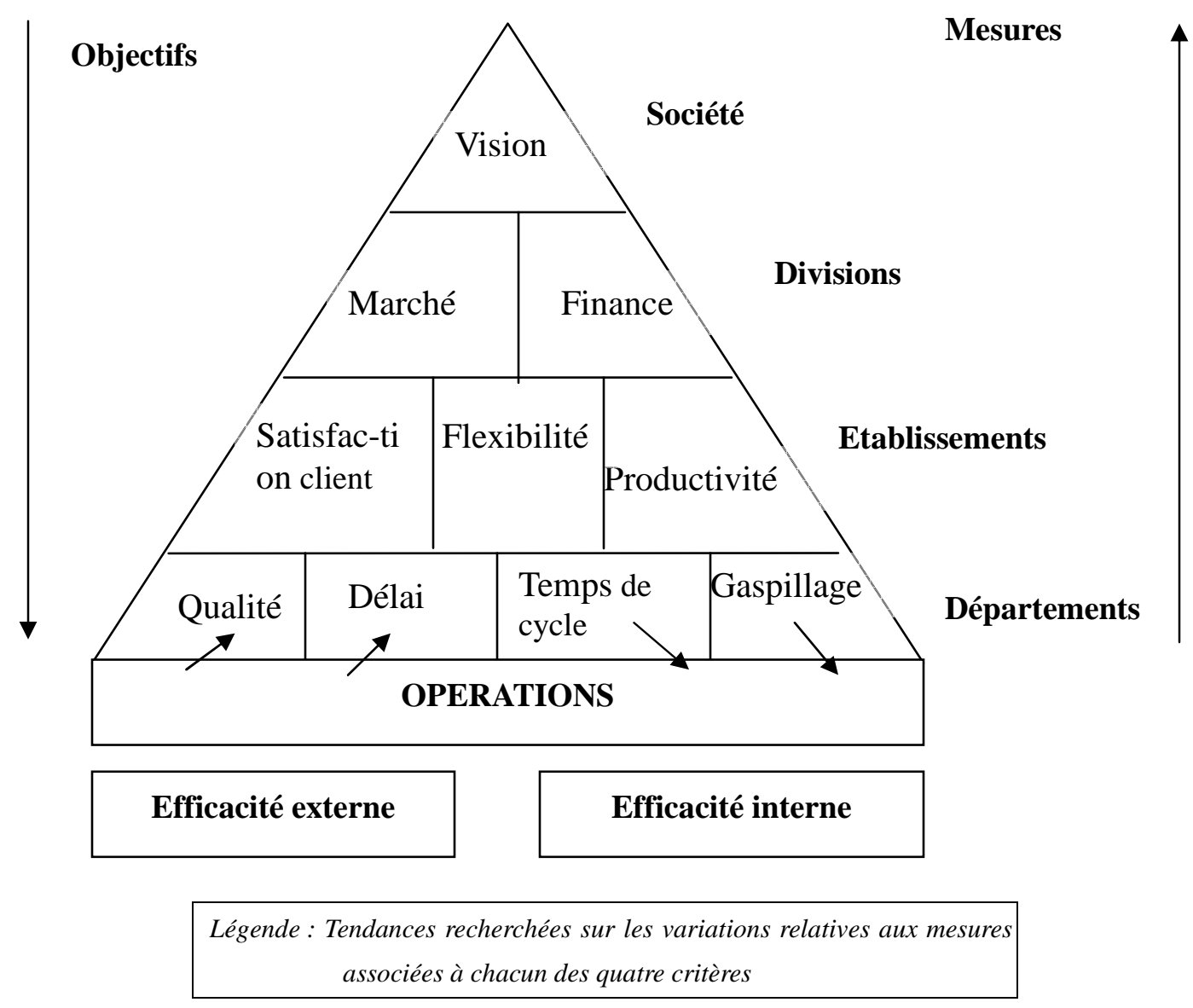

L'objectif de Montebello (1976) est de mettre en valeur les dimensions et les indicateurs qui expliquent l'efficacité organisationnelle. L'annexe 1 récapitule les dimensions et les principaux indicateurs sélectionnés. Dans un premier temps, ils mettent en valeur les trois principales dimensions qui représentent au mieux l'efficacité. Le choix s'articule autour (1) de la valeur organisationnelle, (2) de la capacité d'adaptation et (3) de la capacité de réponse. Cependant, l'auteur note que les deux premières dimensions sont préférées par les managers d'entreprise. Dans un second temps, les dimensions de second et de troisième rang sont corrélées aux trois principales dimensions. Enfin, dans un troisième temps, une liste exhaustive d'indicateurs est proposée. Toutefois, et en regard des réponses fournies par les managers, Montebello (1976) souligne l'existence d'indicateurs «phares» de l'efficacité organisationnelle. Ainsi, (1) la 
croissance des profits nets, (2) la marge nette, (3) le rendement des actifs, (4) la qualité des emplois, (5) la qualité de la direction générale et (6) la satisfaction des salariés constituent les indicateurs majeurs de la valeur organisationnelle. Pour le facteur capacité d'adaptation, les indicateurs choisis sont (1) la participation des managers à la planification, (2) la clarté des définitions des tâches de production, (3) la responsabilité des managers de division et (4) la formalisation du plan à long terme. Enfin, pour le facteur capacité de réponse, deux indicateurs sont sélectionnés soit (1) l'[actif courant-dettes courantes]/actif total et (2) le taux d'absentéisme.

En nous appuyant sur le travail de Montebello, il est envisageable de répartir les indicateurs liés au facteur de $3^{\text {ème }}$ ordre selon une configuration propre à la démarche du Développement Durable. En effet, compte-tenu que certains indicateurs se retrouvent sur les trois facteurs principaux selon la configuration de Montebello, la répartition sur le dernier niveau semble la plus aisée et la plus claire. Il convient également de noter ici la très faible représentation d'indicateurs de type environnemental. 
Tableau 1. L'efficacité organisationnelle de Montebello (1976) vu sous l'angle du Développement Durable (d'après l'approche d'analyse de Morana, 2010)

\begin{tabular}{|c|c|}
\hline \multicolumn{2}{|l|}{ INDICATEURS ECONOMIQUES } \\
\hline $\begin{array}{l}\text { - Actifs courants - dettes courantes / actifs totaux } \\
(\mathbf{- 0 . 5 6} \mathbf{~ p m - P )} \\
\text { - Actifs courants - stocks / dettes courantes } \\
\text { - Actifs courants / dettes courantes } \\
\text { - Action prise lorsque les objectifs ne sont pas atteints } \\
\text { - Chiffre d'affaires / actifs } \\
\text { - Croissance des actifs totaux } \\
\text { - Croissance des coûts des produits vendus } \\
\text { - Croissance des fonds propres } \\
\text { - Croissance des profits nets } \\
\text { - Croissance des ventes }\end{array}$ & 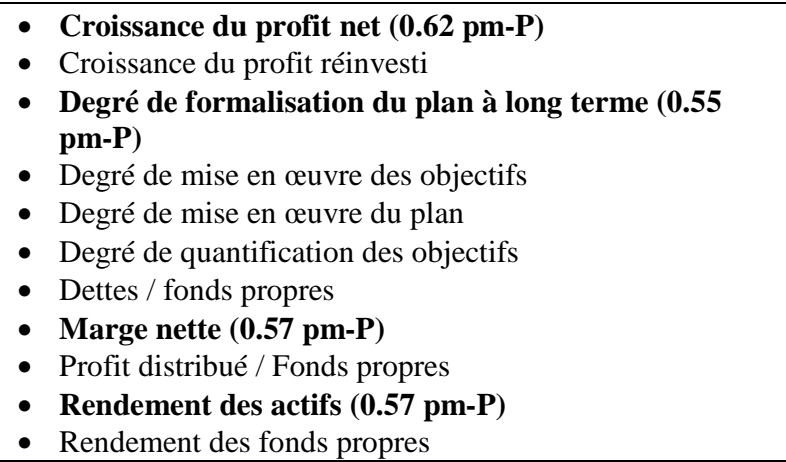 \\
\hline \multicolumn{2}{|l|}{ INDICATEURS ENVIRONNEMENTAUX } \\
\hline \multicolumn{2}{|l|}{$\begin{array}{l}\text { Instabilité de l'environnement } \\
\text { Pollution de l'environnement }\end{array}$} \\
\hline \multicolumn{2}{|l|}{ INDICATEURS SOCIAUX / SOCIETAUX } \\
\hline $\begin{array}{l}\text { - } \text { Association récompense/performance des } \\
\text { directeurs de division } \\
\text { - Autorité financière des responsables de division } \\
\text { - Clarté des définitions des tâches de production } \\
\text { (0.60 pm-P) } \\
\text { - Clarté des définitions des tâches de R\&D } \\
\text { - Communication vers l'amont } \\
\text { - Communication vers l'aval } \\
\text { - Conflits intraorganisationnels } \\
\text { - Connaissance par les salariés des objectifs de leur } \\
\text { - unité de travail } \\
\text { - Contribution fiscale de l'entreprise } \\
\text { - Degré de participation des managers dans la } \\
\text { - } \text { Inanification (0.68 pm-P) } \\
\text { - Nombre de clients indépendants } \\
\text { - Nombre de fournisseurs indépendants } \\
\text { - Nombre de marchés indépendants } \\
\text { - Nombre de nouveaux emplois offerts chaque année } \\
\text { - Participation des salariés dans la prise de décision } \\
\text { - Problèmes de relation de travail }\end{array}$ & $\begin{array}{l}\text { - Qualité de la direction générale (0.55 pm-P) } \\
\text { - Qualité des emplois (0.56 pm-P) } \\
\text { - Qualité et quantité de R\&D } \\
\text { - Réaction aux menaces perçues } \\
\text { - Réaction aux opportunités perçues } \\
\text { - Récompense en fonction du niveau de réalisation des } \\
\text { - } \text { objectifs } \\
\text { - Responsabilité de planification des directeurs de } \\
\text { - Responsabilité des résultats de la division } \\
\text { - Responsabilité des résultats fonction des responsabilités de } \\
\text { - Slanification } \\
\text { - Salaires moyens dans l'entreprise } \\
\text { - Stabilité de la direction générale } \\
\text { - Taux d'absentéisme (-0.55 pm-P) } \\
\text { - Taux de rotation des salariés } \\
\text { - Utilisation des capacités des salariés }\end{array}$ \\
\hline
\end{tabular}




\section{La notion de performance logistique}

Si la performance logistique doit s'inscrire dans une évaluation de type processuel, à travers -par exemple- la méthode de l'Activity Based Costing ou la démarche de l'Efficient Consumer Response, la littérature portant sur l'évaluation de la performance de la logistique tend à montrer par ailleurs que celle-ci peut se fonder sur l'élaboration d'outils d'aide à la décision tels que les tableaux de bord (Kueng, 2000 ; Gunasekaran et al., 2001 ; Otto et Kotzab, 2001a,b), outils qui mettent en exergue des indicateurs de type qualitatif et quantitatif.

Pour Chow et al. (1994), "la performance logistique peut être vue comme un sous-élément de la notion élargie de performance de la firme ou de l'organisation». Selon ces auteurs, la "performance de la logistique peut être définie comme l'extension de chaque objectif achevé » (p. 23) comme ceux suggérés dans la Figure 3 ci-dessous :

Figure 2. Les différentes façons d'envisager la performance logistique (selon Chowet al., 1994)

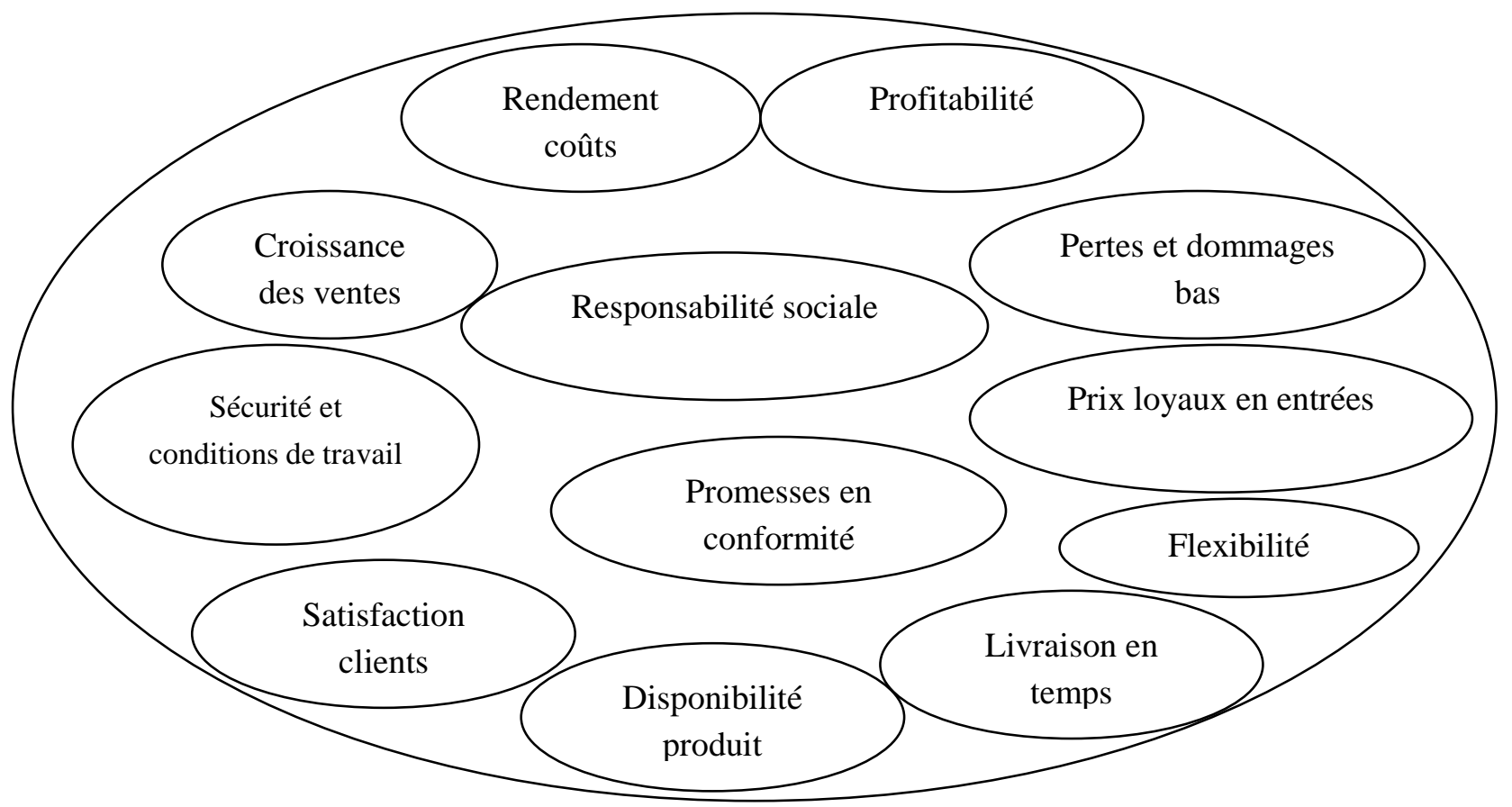

Chow et al. (1994) définissent la performance logistique à travers l'adjonction d'éléments de nature hard (revenus nets ou chiffres comptables) et soft (taux de satisfaction de la clientèle). En 
fonction de quoi, celle-ci permet de déterminer un ensemble de dimensions à court et long horizons capables de mesurer l'apport de plusieurs organisations, et de reconnaittre les relations de chaque membre au sein d'un SCM.

Suivant cette définition, Caplice et Sheffi (1994) mettent à jour les critères d'évaluation d'une performance logistique. La métrique ainsi choisie doit être constituée de huit critères qui présentent un caractère

(1) de validité, reflet et contrôle précis des événements et des activités,

(2) de robustesse, correctement interprétée par tous les acteurs et répétée à travers le temps, le lieu et les organisations,

(3) d'utilité, apte à faire comprendre et à fournir un guide pour toutes les actions et décisions entreprises,

(4) d'intégration de tous les composants et aspects des processus dans et hors de la firme,

(5) d'économie à retracer aisément et fidèlement les coûts représentatifs,

(6) de compatibilité avec les systèmes comptables et d'informations détenus par la firme,

(7) de niveau de détail suffisamment clair et explicite à l'utilisateur, et

(8) de neutralité comportementale en vue de minimiser les actes ou jeux individuels et improductifs.

En bref, la mesure de la performance logistique ambitionne une amélioration permanente qui tient compte de ces divers éléments et engendre la conceptualisation et la mise en œuvre de systèmes de mesure qui allient diagnostic et aide à la décision.

Si l'on s'intéresse à l'évaluation du SCM, trois référentiels sont appréciés dans les principaux travaux dans la matière :

- le travail de Gunasekaran et al. (2001). Ils proposent une liste 42 indicateurs, classée en stratégique, tactique et opérationnel. Ce travail est la base de réflexions pratiques (Morana, 2002 ; Bhagwat et Sharma, 2007a,b ; Sharma et Bhagwat, 2007) ;

- l'approche de Gunasekaran and Kobu (2007) avec une liste de 26 indicateurs,

- le travail de Griffis et al. (2007) avec 14 indicateurs.

Si l'on prend en compte les travaux les moins anciens, à savoir Gunasekaran et Kobu (2007) et Griffis et al. (2007), 40 indicateurs seraient représentatifs du SCM. Il convient toutefois de noter que leur mise en commun ne montre pas de convergence. 
Tableau 2. Les indicateurs phares de la logistique (Source : adapté de Gunasekaran et Kobu, 2007 et Griffis et alii, 2007)

\begin{tabular}{|l|l|}
\hline INDICATEURS ECONOMIQUES (coût / qualité / délai) (nombre = 33) \\
\hline Précision de l'ordonnancement ; & Coûts d'obsolescence ; \\
Temps moyen de remplissage des commandes en & Pourcentage de livraison dans les délais ; \\
souffrance ; & Variabilité du temps de cycle de la commande ; \\
Taux moyen de remplissage par ligne de commande ; & Temps de cycle du process ; \\
Temps moyen de cycle de commande ; & Temps de développement du produit ; \\
Temps de cycle de gestion de l'offre ; & Variété des produits/services ; \\
Utilisation des capacités ; & Flexibilité de la production ; \\
Taux de remplissage de la commande complète ; & Retour sur investissement ; \\
Jours de retard sur la commande ; & Pertes de ventes dues aux ruptures de stock ; \\
Fiabilité de la livraison ; & Prix de vente ; \\
Précision des prévisions ; & Coût de rupture ; \\
Coût des inventaires ; & Temps de réponse de la chaîne logistique ; \\
Taux de rotation des inventaires ; & Coût du transport ; \\
Délai d'approvisionnement ; & Valeur ajoutée ; \\
Délai de production ; & Semaines d'approvisionnement ; \\
Ratio coûts logistiques sur ventes ; & Coût de garantie ; \\
Coûts logistiques par unité ; & Frais généraux. \\
\hline INDICATEURS ENVIRONNEMENTAUX \\
\hline Non énumérés \\
\hline INDICATEURS SOCIAUX / SOCIETAUX (nombre = 7) \\
\hline Conformité aux spécifications ; & Qualité perçue ; \\
Conformité aux règlementations ; \\
Articles prélevés par personne et par heure ; \\
Efficacité du travail.
\end{tabular}

Légende : en foncé, les indicateurs économiques de nature quantitative, issus des documents comptables.

Ce panorama non exhaustif sur l'utilisation d'indicateurs au sein des entreprises et dans une perspective logistique va -selon nous- dans le sens des contraintes d'évaluation imposées par les entreprises. En effet, nous retrouvons en priorité les problématiques économiques à-travers la gestion des coûts et par extension la gestion de la qualité et des délais, et les problématiques sociales et sociales dont on peut apprécier la valeur à-travers le bilan social et le bilan sociétal ${ }^{1}$. Pour les indicateurs environnementaux, nous trouvons une énumération générale dans le travail de Depoers et al. (2003) dont nous ne précisons pas ici les intitulés car se positionnant, selon nous, plus sur un plan d'économie locale que sur un plan entrepreneurial.

\footnotetext{
${ }^{1}$ Pour rappel, le bilan social est obligatoire depuis la loi du 12 juillet 1977 dans les entreprises de plus de 300 salariés et le bilan sociétal, bien que non obligatoire, existe depuis 1996 (Source : http://www.cjdes.org).
} 


\section{Les indicateurs de durabilité de la logistique urbaine}

Si la logistique globale suit des principes d'entreprise, la logistique urbaine est en général liée aux actions de plusieurs acteurs, et les visions "entreprise" sont confrontées aux visions "collectivités", i.e., aux actions et objectifs des pouvoirs publics. Il a été constaté que les projets de logistique concernent des secteurs très divers qui soulèvent des questions en termes de faisabilité, d'acceptabilité et d'impact de natures très différentes. Il est donc important de tenir compte de ces secteurs dans la recherche d'indicateurs de performance et choisir ceux qui répondent aux besoins et objectifs de chacune des parties. Ces indicateurs sont parfois difficilement mesurables ou accessibles.

La chaîne d'évaluation d'un projet peut se décliner du processus de sa conception, de son démarrage (évaluation ex ante), de son suivi (monitoring) jusqu'à son évaluation ex post comme dans le schéma suivant :

Figure 3. Les différentes phases d'évaluation d'un projet d'espace logistique urbaine (selon Boudouin, 2006, p. 72)
(1) Préparation du projet
(2) Expérimentation
(3) Pérennisation

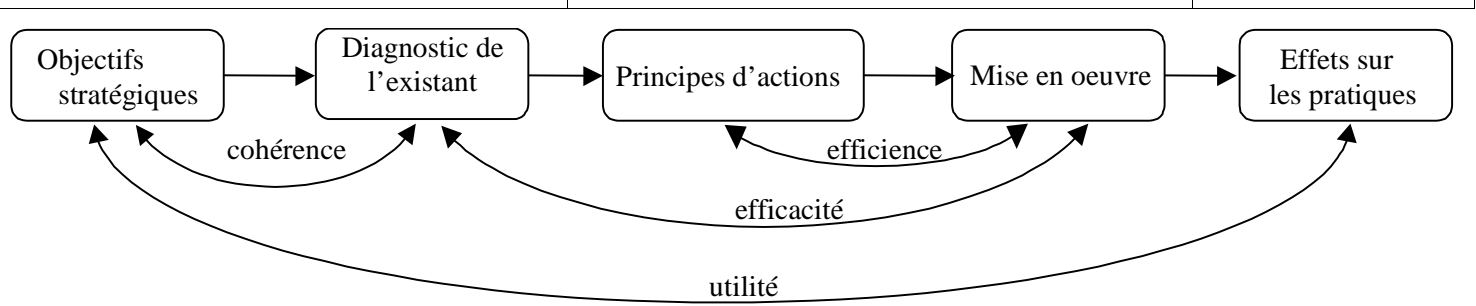

Les relations symbolisées par les flèches dans le schéma doivent satisfaire aux critères de cohérence, d'efficacité, d'efficience et d'utilité.

Les indicateurs traditionnels des transports de marchandises de longue distance (tonnes transportées, tonnes*km, quantité d'énergie consommée par tonne*km, km parcourus à vide, etc.) semblent peu pertinents au niveau urbain. Par exemple, transporter une tonne de marchandise donnée dans la ville peut être réalisé de multiples façons (selon le nombre d'envois, la taille des véhicules, le nombre de colis), variété qui ne peut être appréhendée qu'à travers une étude approfondie de la génération des livraisons et des organisations correspondantes. Il en va de même de la mesure du retour à vide dont le calcul nécessite de connaître le diagramme de charge de chaque véhicule. A partir des enquêtes nationales «Marchandises en Ville», plusieurs indicateurs pour le diagnostic du transport de marchandises en ville peuvent être calculés (Patier 
et Routhier, 2009). Le tableau 4 montre la richesse des indicateurs qui peuvent être calculés grâce aux Marchandises en Ville. Comme indiqué dans la troisième colonne, chaque indicateur aide à mesurer l'implication des différents secteurs d'activités sur les mouvements de marchandises en ville et apporte de précieuses informations sur la part des modes de gestion (compte propre, compte d'autrui) et d'organisation (taille des véhicules, taille des tournées). 
Tableau 1 : Les indicateurs produits par les enquêtes TMV (Patier et Routhier, 2009)

\begin{tabular}{|c|c|c|}
\hline $\begin{array}{l}\text { Nom et description de } \\
\text { l'indicateur }\end{array}$ & $\begin{array}{l}\text { Unité de mesure de } \\
\text { l'indicateur }\end{array}$ & $\begin{array}{l}\text { Objet de l'indicateur } \\
\text { Permet mesures : }\end{array}$ \\
\hline $\begin{array}{l}\text { Ratio de livraisons/enlèvements } \\
\text { par emploi dans un secteur } \\
\text { d'activité }\end{array}$ & $\begin{array}{l}\text { Nombre de } \\
\text { livraisons/enlèvements par } \\
\text { semaine par employé. }\end{array}$ & $\begin{array}{l}\text { Rapide évaluation de la génération des } \\
\text { livraisons/enlèvements dans la ville sans } \\
\text { nouvelle enquête. La contribution de chaque } \\
\text { secteur d'activité est calculable. }\end{array}$ \\
\hline $\begin{array}{l}\text { Densité de } \\
\text { livraisons/enlèvements dans une } \\
\text { zone }\end{array}$ & $\begin{array}{l}\text { Nombre de } \\
\text { livraisons/enlèvements par } \\
\text { semaine par } \mathrm{km}^{2}\end{array}$ & $\begin{array}{l}\text { Importance des flux de véhicules de } \\
\text { marchandises dans une zone. }\end{array}$ \\
\hline $\begin{array}{l}\text { Intensité de } \\
\text { livraisons/enlèvements d'un } \\
\text { secteur d'activité dans une zone }\end{array}$ & $\begin{array}{l}\text { Nombre de } \\
\text { Livraisons/enlèvements par } \\
\text { semaine. }\end{array}$ & $\begin{array}{l}\text { Contribution de chaque secteur d'activité au } \\
\text { trafic de marchandises en ville par zone. }\end{array}$ \\
\hline $\begin{array}{l}\text { Durée de livraisons/enlèvements } \\
\text { en double file pour livrer ou } \\
\text { enlever un secteur d'activité dans } \\
\text { une zone. }\end{array}$ & Nombre d'heures & $\begin{array}{l}\text { Contribution de chaque secteur d'activité à la } \\
\text { congestion de la voirie par le stationnement sur } \\
\text { voirie en double file par zone. }\end{array}$ \\
\hline $\begin{array}{l}\text { Distance moyenne parcourue } \\
\text { pour livrer ou enlever un secteur } \\
\text { d'activité avec un type de } \\
\text { véhicule. }\end{array}$ & Nombre de km & $\begin{array}{l}\text { Contribution des différents types de véhicules } \\
\text { qui livrent chaque secteur d'activité à la } \\
\text { congestion de la voirie. }\end{array}$ \\
\hline $\begin{array}{l}\text { Longueur de la marche } \\
\text { d'approche de la plate forme au } \\
\text { lieu de livraison }\end{array}$ & Nombre de km. & $\begin{array}{l}\text { Impact du lieu d'implantation de la plate-forme } \\
\text { livrant les produits concernant son aire de } \\
\text { marché. }\end{array}$ \\
\hline $\begin{array}{l}\text { Distance moyenne nécessaire } \\
\text { pour livrer/enlever }\end{array}$ & $\begin{array}{l}\text { km par } \\
\text { livraisons/enlèvements }\end{array}$ & $\begin{array}{l}\text { Contribution d'un Livraisons/enlèvements au } \\
\text { trafic urbain (par type de véhicule impliqué). }\end{array}$ \\
\hline $\begin{array}{l}\text { Distance totale parcourue dans } \\
\text { l'aire urbaine par les véhicules de } \\
\text { marchandises }\end{array}$ & véhicules*km par semaine & $\begin{array}{l}\text { Contribution de l'activité totale à la génération } \\
\text { du trafic motorisé urbain. }\end{array}$ \\
\hline $\begin{array}{l}\text { Durée moyenne d'une } \\
\text { livraison/enlèvement (par secteur } \\
\text { d'activité, type de véhicule, mode } \\
\text { de gestion) }\end{array}$ & Minutes par livraison & $\begin{array}{l}\text { Temps de livraison lors d'un parcours, sur la } \\
\text { route, pour un secteur d'activité, un type de } \\
\text { véhicule, nu mode de gestion donné. }\end{array}$ \\
\hline $\begin{array}{l}\text { Vitesse moyenne par tournée } \\
\text { (comprenant ou excluant les } \\
\text { arrêts pour livrer). }\end{array}$ & $\mathrm{Km} / \mathrm{h}$ & $\begin{array}{l}\text { Performance des tournées pour chaque mode } \\
\text { d'organisation, type de véhicule. }\end{array}$ \\
\hline $\begin{array}{l}\text { Poids transporté au kilomètre par } \\
\text { trajet selon le secteur d'activité, } \\
\text { le type de véhicule, le mode de } \\
\text { gestion }\end{array}$ & Tonne (ou kg)*km & $\begin{array}{l}\text { Performance des tournées pour chaque mode } \\
\text { d'organisation, type de véhicule... }\end{array}$ \\
\hline $\begin{array}{l}\text { Consommation d'énergie, } \\
\text { pollution, effet de serre selon la } \\
\text { zone, le véhicule, l'activité, le } \\
\text { mode de gestion (totale ou par } \\
\text { mouvement) }\end{array}$ & $\begin{array}{l}\text { - tonnes de Gazole. } \\
\text { - kg Polluant (Nox, SO2, CO, } \\
\mathrm{PM}, \ldots) \\
\text { - } \mathrm{kg} \mathrm{CO} 2\end{array}$ & $\begin{array}{l}\text { Impact des mouvements de marchandises sur la } \\
\text { consommation d'énergie, les nuisance locales } \\
\text { et globales et les gaz à effets de serre. }\end{array}$ \\
\hline
\end{tabular}


Henriot et al. (2008) identifient une série d'indicateurs pour l'évaluation de projets de logistique urbaine. Cinq grandes catégories d'indicateurs ont été retenues comme fondamentales dans l'évaluation de ce type de projets, et sont les suivantes :

- Economique et commercial : indicateurs macroscopiques de performance (principalement de type économique) du point de vue du porteur du projet en tant qu'entrepreneur. Elles conditionnent la continuité du projet et donc ses possibilités de réussite. Les variables à être privilégiées du point de vue strictement économique sont principalement :

1. Les montants bruts des investissements (hors taxes),

2. Les charges d'exploitation (selon les normes du Plan Comptable),

3. Les subventions - avances remboursables, soutiens directs et indirects reçus,

4. L'adéquation des tarifs des prestations réalisées aux standards du marché,

5. Le chiffre d'affaires réalisé.

6. La satisfaction des clients/usagers (mesurée par enquête de satisfaction).

7. La "visibilité" du projet sur le plan médiatique (mesurée par un taux de notoriété).

Technique et logistique : indicateurs macroscopiques de performance (principalement de type économique) plus orientés sur les aspects organisationnels et opérationnels du projet. Il est important de se faire une idée précise de la performance logistique (avec des variables similaires à celles présentées en section 0). Les principales variables dans ce groupe sont les suivantes :

1. nombre de colis/palettes livrés ou enlevés,

2. nombre de positions, d'arrêts,

3. durée des arrêts,

4. zone de chalandise,

5. distance sur mode routier classique,

6. capacité des véhicules,

7. \% de charge de la plate-forme logisitque,

8. \% d'utilisation,

9. nombre de camions accueillis sur la plate-forme.

Social et ergonomique : ces deux dimensions ont été considérées dans le même groupe car elles sont strictement liées. Les trois principales variables sont respectivement les conditions de travail - ergonomie du projet, le nombre d'emplois et la formation - insertion respectivement.

Environnemental : ces indicateurs sont groupés en quatre catégories, qui sont les suivantes :

1. variation de la consommation énergétique (cet indicateur a aussi de plus en plus une dimension économique),

2. variation des émissions de polluants $\left(\mathrm{CO}_{2}, \mathrm{NO}_{\mathrm{X}}, \mathrm{SO}_{\mathrm{X}}, \mathrm{VOC}, \mathrm{PM} 10, \ldots\right)$,

3. part de la distribution effectuée avec des véhicules propres (en vehicules.km et, si 
possible en tonnes.km ),

4. émissions de polluants, (ces données peuvent être aussi utiles sous forme de valeur économique).

Réglementation - occupation de l'espace public : indicateurs de performance globale de la logistique urbaine du point de vue de la collectivité. Il est important dans ce groupe de tenir compte des conditions réelles d'occupation de l'espace et du respect de la réglementation en matière de circulation et de stationnement. Les variables retenues sont les suivantes :

1. la part des livraisons interdites/autorisées,

2. l'encombrement de la voirie,

3. la durée de stationnement gênant.

D'autres indicateurs, dits complémentaires, peuvent être définis. Ces indicateurs ont été groupes en suivant la même catégorisation que pour ceux considérés comme fondamentaux. Cette méthodologie, très détaillée, reste difficile à mettre en œuvre de façon opérationnelle, d'autant plus qu'elle ne permet pas une vraie comparaison entre expériences (car elle n'oblige pas à utiliser les mêmes indicateurs) mais une comparaison entre méthodes d'évaluation. Un exemple de cette limite peut être observé dans Patier et Browne (2010). Néanmoins, la liste d'indicateurs reste une base intéressante à garder et combiner avec d'autres travaux contemporains, pour définir un socle d'indicateurs qui ressortent de façon récurrente en littérature. Il est important de signaler ici les travaux de Melo (Melo et Costa, 2007, 2011; Melo, 2010) qui, en parallèle à Henriot et al. (2008) définit une liste d'indicateurs plus ample mais non hiérarchisée. Si bien on retrouve beaucoup d'indicateurs en commun, les travaux de Melo permettent d'introduire des indicateurs classiques du transport de marchandises qui, bien que non tous adaptés à la nature urbaine, doivent être considérés dans l'interface entre transport urbain et transport interurbain de marchandises.

D'autres travaux s'adressent à la durabilité en logistique urbaine. Behrends et al. (2008) cherchent à définir un indicateur unique de durabilité. Taniguchi et Van Der Heijden (2000), Gonzalez-Feliu (2008) et Vaghi et Percoco (2011) identifient différents éléments sur les nuisances principalement environnementales, comme les émissions de polluants, mais aussi la congestion et le bruit. Morana et Gonzalez-Feliu (2010) s'intéressent plutôt aux aspects économiques mais aussi sociétaux, compte tenu des principaux indicateurs environnementaux attendus en logistique urbaine, pour le CDU de Padoue. Les auteurs réalisent une analyse qualitative de la durabilité en identifiant les principaux facteurs de succès, qui sont principalement d'ordre économique. De cette liste non exhaustive de travaux, nous constatons un manque d'unification et de standards qui rendent difficile la comparaison entre expériences de logistique urbaine. D'ailleurs, comme le soulignent Morana et Gonzalez-Feliu (2011), les méthodes existantes s'adressent principalement aux pouvoirs publics mais, pour faire fonctionner un système mutualisé de livraisons, il faut 
l'accord et la collaboration de tous ses acteurs, principalement les privés (Gonzalez-Feliu et Morana, 2010). De plus, la dimension globale de la chaîne logistique et son interface avec le transport de marchandises sont souvent négligés.

\section{Conclusion}

L'évaluation des projets de logistique urbaine doit se positionner dans une perspective de développement durable. Dans ce sens, les trois dimensions (économique, environnementale et sociale) doivent être prises en compte. De plus, les spécificités de la logistique urbaine, et les deux visions prédominantes (entreprise et collectivité publique respectivement) dans les problématiques de mobilité de marchandises dans les zones urbaines font de l'évaluation et la valorisation des projets de logistique urbaine un domaine qui présente un fort potentiel.

Du point de vue économique, la vision entreprise doit être prédominante. Deux grands groupes d'indicateurs sont jugés dans les différents travaux (tant sur la logistique globale comme sur le transport de marchandises en ville) : les indicateurs macroscopiques de continuité économique de l'entreprise et les indicateurs de performance économique logistique. En ce qui concerne la dimension environnementale, les principales variables à étudier sont les suivantes: la consommation énergétique, les variations des émissions de polluants par rapport à une situation initiale et par rapport à l'ensemble des émissions pour le transport urbain (personnes + marchandises). La dimension sociale est plus difficile à caractériser et nécessite d'une étude plus approfondie. Néanmoins, la valorisation sociale à l'intérieur de l'entreprise, les variations des emplois et la réaffectation d'emplois semblent les principales variables dans la recherche d'indicateurs sociaux et sociétaux. Finalement, les questions liées à la congestion et au respect des réglementations sont aussi à considérer, dans une approche systémique de la logistique urbaine.

\section{Références}

[1] Behrends, S., Lindholm, M., \& Woxenius, J. (2008). The impact of urban freight transport: A definition of sustainability from an actor's perspective. Transportation planning and technology, 31(6), pp. 693-713.

[2] Bhagwat, R., Sharma, M. (2007a), Performance measurement of supply chain management: A balanced scorecard approach, Computers \& Industrial Engineering, 53(1), pp. 43-62. 
[3] Bhagwat, R., Sharma, M. (2007b), Performance measurement of supply chain management using the analytical hierarchy process, Production Planning \& Control, 18(8), pp. 666-680.

[4] Boudouin, D. (2006). Guide de mise en xuvre d'espaces logistiques urbains. CRET-Log, Action fédérative ELU, PREDIT.

[5] Bouquin, H. (1997), Les fondements du contrôle de gestion, Presses Universitaires de France, Paris, $2^{\text {ème }}$ éd.

[6] Bourrier, J., Guillot, J. M., \&Locherer, C. (1998). Mise en place d'un systeme d'indicateurs de performances. Revue Française de Gestion Industrielle, pp. 23-42.

[7] Caplice, C., Sheffi, Y. (1994). A review and evaluation of logistics metrics?. The International Journal of Logistics Management, 5(2), pp. 11-28.

[8] Chow, G., Heaver, T. D., \& Henriksson, L. E. (1994). Logistics performance: definition and measurement. International journal of physical distribution \& logistics management, 24(1), 17-28.

[9] Cross, K., Lynch, R. (1989). Accounting for competitive performance. Journal of Cost Management, 3(1), pp. 20-28.

[10] Dablanc, L. (1998). Transport de marchandises en ville. Une gestion publique entre police et services. Liaisons, Paris.

[11] Depoers, F., Reynaud, E., \& Maunoury, G. S. (2003). Indicateurs de développement durable. CER2D, Observatoire des stratégies industrielles.

[12] Gonzalez-Feliu, J. (2008), Models and Methods for the City Logistics. The Two-echelon Capacitated Vehicle Routing Problem. PhD. Thesis. Politecnico di Torino, Turin, Italy.

[13] Gonzalez-Feliu, J., Morana, J. (2010), A la recherche d'une mutualisation des livraisons en milieu urbain : le cas du groupe NMPP, Revue Française de Gestion Industrielle, vol 29, n.2, pp. 71-92.

[14] Griffis, S.E., Cooper, M., Goldsby, T.J., Closs, D.J. (2004). Performance measurement: measure selection based upon firm goals and information reporting needs, Journal of Business Logistics, 25(2), pp. 95-118.

[15] Griffis, S.E., Goldsby, T.J., Cooper, M., Closs, D.J. (2007). Aligning logistics performance measures to the information needs of the firm, Journal of Business Logistics, 28(2), pp. $35-56$.

[16] Gunasekaran, A. And Kobu, B. (2007), Performance measures and metrics in logistics and supply chain management: a review of recent literature (1995-2004) for research and applications, International Journal of Production Research, 45(12), pp. 2819-40.

[17] Gunasekaran, A., Patel, C. Tirtiroglu, E. (2001), Performance measures and metrics in a 
supply chain environment, International Journal of Operations \& Production Management, 21 (1-2), pp. 71-87.

[18] Henriot, F., Patier, D., Bossin, P., Gérardin B. (2008). Méthodologie d'évaluation des innovations en matière de logistique urbaine, rapport PREDIT-DRAST.

[19] Kaplan, R., Norton, D. (1996c), The balanced scorecard, Harvard Business School Press, Boston (MA).

[20] Kueng, P. (2000). Process performance measurement system: a tool to support process-based organizations. Total Quality Management, 11(1), pp. 67-85.

[21] Marmuse, C. (1997). Performance. In Joffre, P. et Simon, Y. (coord.), Encyclopedie de gestion, pp. 2194-2208.

[22] Melo, S. (2010). Evaluation of urban goods distribution initiatives towards mobility and sustainability indicators, stakeholders and assessment tools. PhD. Thesis, University of Porto, Portugal.

[23] Melo, S., \& Costa, A. (2011). Definition of a set of indicators to evaluate the performance of urban goods distribution initiatives. In Macharis, C., Melo, S. (eds.), City Distribution and Urban Freight Transport: Multiple Perspectives. Edward Elgar, Northampton, pp. to appear.

[24] Melo, S., Costa, A. (2007), Effects of collaborative systems on urban distribution. Proceedings of the 11th World Conference on Transport Research, Berkeley, USA, June.

[25] Montebello, M.H. (1976). Efficacité de l'entreprise: analyse et perspectives, Thèse de doctorat.

[26] Morana, J. (2002). Le couplage supply chain management - tableau de bord stratégique : une approche exploratoire, Thèse en Sciences de Gestion, Université d'Aix-Marseille II.

[27] Morana, J. (2010). Le Sustainable Supply Chain Management: une première étape de modélisation. $8^{\text {èmes }}$ Rencontres Internationales de Recherche en Logistique (RIRL), Bordeaux, septembre.

[28] Morana, J., Gonzalez-Feliu, J. (2010). Sustainable supply chain management in city logistics solutions: lessons learned from the case of Cityporto Padua (Italy). In Proceedings of the 3 rd International Conference on Information Systems, Logistics and Supply Chain, April 14-16, 2010, Casablanca (Moroco).

[29] Morana, J., Gonzalez-Feliu, J. (2011). Le transport vert de marchandises : l'expérience de la ville de Padoue en Italie, Gestion : Revue Internationale de Gestion, à paraître.

[30] Otto, A., Kotzab, H. (2001a), Does supply chain management really pay? Six perspectives to measure the performance of managing a supply chain, Proceedings of the 13th Annual 
NOFOMA Conference, Reykjavik, June, disponible sur http://www.nofoma.org.

[31] Otto, A., Kotzab, H. (2001b), Perspective-driven performance measurement for supply chain management, Logistics Research Network Conference Proceedings, Edinburgh, September, CD-Rom.

[32] Patier, D., \& Browne, M. (2010). A methodology for the evaluation of urban logistics innovations. Procedia-Social and Behavioral Sciences, 2(3), pp. 6229-6241.

[33] Patier, D., Routhier, J.L. (2009), Une méthode d'enquête du transport de marchandises en ville pour un diagnostic en politiques urbaines, Les Cahiers Scientifiques du Transport, 55, pp. 11-38.

[34] Sharma, M., Bhagwat, R. (2007), An integrated BSC-AHP approach for supply chain management evaluation, Measuring Business Excellence, 11(3), pp. 57-68.

[35] Srivastava, S. (2007), Green supply-chain management: A state-of-the-art literature review, International Journal of Management Reviews, 9 (1), pp. 53-80.

[36] Taniguchi, E., Van Der Heijden, R. E. (2000). An evaluation methodology for city logistics. Transport Reviews, 20(1), pp. 65-90.

[37] Vaghi, C., Percoco, M. (2011). City Logistics in Italy: success factors and environmental performance. In Macharis, C., Melo, S. (eds.), City Distribution and Urban Freight Transport: Multiple Perspectives. Edward Elgar, Northampton, to appear. 